\title{
Österreichische Periodika in französischer Sprache als Medien des Literaturtransfers (1750-1850)
}

\begin{abstract}
Der Beitrag präsentiert am Beispiel von vier in Wien erschienenen frankophonen Periodika, auf welche Weise, in welchem Umfang und mit welchem Ziel französische Literatur zwischen 1750 und 1850 nach Österreich vermittelt wurde. Nicht weniger als 97 - oft kurzlebige - Zeitschriften liefern vor allem dem mittleren bis höheren Bürgertum, bei dem es sich in dem behandelten Zeitraum um noch kein allzu umfangreiches Publikum handelt, Informationen über die Kultur der République des Lettres. Anhand der Inhalte des Journal de Vienne, dédié aux amateurs de la litterature (1784-85) aus der josephinischen Ära, der Anthologie littéraire et universelle (1805-06) aus der Phase der napoleonischen Kriege, des Spectateur. Journal historique, littéraire, moral, politique et dramatique (1819) aus der Zeit des Kaisertums unter Franz I. und des Journal de la littérature étrangère (1841) aus der Epoche des Vormärz werden Gestalt und Bedeutung von Periodika für den französisch-österreichischen Kulturaustausch in einer von historischen Diskontinuitäten geprägten Epoche stichprobenartig beleuchtet.
\end{abstract}

Keywords: Zeitschriften, Literaturtransfer, Österreich, Frankreich

\begin{abstract}
Um die Mitte des vorigen Jahrhunderts war Paris die Hauptstadt Europa's. Als Voltaire in Potsdam wohnte, meinte er in Frankreich zu sein. Wofür Ludwig XIV. seine Generäle, seine Armeen vergebens in das Feld geschickt hatte, das war für die Denker und Dichter, für die geistreichen Frauen Frankreichs die Frucht leichter Siege. Die Universalmonarchie des Geistes war gegründet, während die politische ein Phantom blieb, das noch einige Zeit die Völker schrecken sollte.

Leopold von Sacher-Masoch, Kaunitz (1873)
\end{abstract}

Als der österreichische Staatskanzler Kaunitz, „a peculiarly francophile mind“ (Szabo 1994, 34), ${ }^{1}$ das im Vorfeld des Siebenjährigen Krieges geschlossene Defensivbündnis gegen Preußen und England (1756) in die Wege leitet, bereitet er dem beinahe 250 Jahre währenden habsburgisch-französischen Gegensatz ein Ende.

1 Kaunitz, zuvor Gesandter in Paris, ist mit französischen Bühnenwerken des siebzehnten und achtzehnten Jahrhunderts bestens vertraut und ein ,avid collector of French books, one of the first subscribers to the Encyclopédie and an enthusiastic reader of Voltaire“ (Szabo 1994, 33), daher der Ruf eines „Gallomanen“ (Wagner 1961, 509). 
Mit der Überwindung der Rivalität um die Hegemonie in Europa durch dieses renversement des alliances rücken die Länder auch kulturell zusammen, was ein erheblicher Anstieg von Transferaktivitäten belegt. Zwar reichen die Kulturkontakte bis ins Mittelalter zurück (vgl. Fichtenau 1962), ein institutionell vermittelter Transfer substantiellen Ausmaßes ist jedoch erst seit Mitte des achtzehnten Jahrhunderts $\mathrm{zu}$ verzeichnen. Er weist en gros eine unilaterale Ausrichtung auf: Frankreich ist nicht nur in politischer Hinsicht „Experimentierlabor der neueren europäischen Geschichte“ (Wehler 2005, 345), auch seine Originalität in künstlerischen Belangen liefert traditionsmüden Geistern in kulturell konservativeren Ländern wie Österreich Inspirationen.

Ideen, weiß Philipp Joseph Schalbacher, einer der führenden Vermittler fremdsprachiger Texte im deutschsprachigen Raum (vgl. Frank und Frimmel 2008, 164), zirkulieren vor allem „par la conversation, par les livres et par les journaux“ [über Gespräche, über Bücher und über Zeitschriften] (Anthologie 1805 [Bd. 2, Nr. 2] 126). In Österreich erscheinen zwischen 1750 und 1850 insgesamt 97 Periodika in französischer Sprache, ${ }^{2}$ von denen im Folgenden vier Literaturzeitschriften aus verschiedenen Jahrzehnten herangezogen werden, um die Kulturvermittlung in dieser durch starke Diskontinuitäten geprägten Epoche zu skizzieren: das Journal de Vienne (1784-1785), die Anthologie littéraire et universelle (1805-1806), Le Spectateur (1819) und das Journal de la littérature étrangère (1841). Die kulturelle Hegemonie Frankreichs veranschaulicht bereits die Tatsache, dass den 97 frankophonen Zeitschriften im selben Zeitraum insgesamt 94 anderssprachige gegenüberstehen, darunter italienische (21), ungarische (19), griechische (16), lateinische (7) und tschechische (6). Unter den 97 französischsprachigen Periodika lassen sich 17 primär dem literarischen Bereich zuordnen (mit Besprechungen, Literaturgeschichtlichem, Lyrik, Erzählungen, Roman- und Dramenauszügen als überwiegendem Inhalt). An oberster Stelle in thematischer Hinsicht stehen 21 Musik-Periodika, bei denen es sich allerdings vornehmlich um Notenmaterial handelt. Ein dritter hervorhebenswerter Themenbereich umfasst dem Spracherwerb und der Erziehung dienende Zeitschriften. Während unter den Jugend- und Musikzeitschriften einige die Fünf-Jahres-Grenze überdauern, werden die meisten der 17 Literatur-

2 Diese auch im folgenden verwendeten Angaben stellen eine Auswertung der Datensätze aus der Österreichischen Retrospektiven Bibliographie (ORBI) dar (vgl. Lang und Lang 2006). Bei einigen Zeitschriften von kurzer Erscheinungsdauer hat sich nur ein bibliographischer Vermerk erhalten, aber kein Exemplar, so dass die tatsächliche Veröffentlichung fraglich ist. Während manche deutschsprachige Periodika in anderen Städten des Habsburgerreiches (Prag, Pressburg, Brünn) erscheinen, werden die frankophonen (mit Ausnahme des in Pest verlegten und in Wien gedruckten Le nouvelliste français, 1815-1816) in Wien, Residenzstadt und Zentrum der Diplomatie, herausgegeben. 
zeitschriften innerhalb eines Jahres, spätestens innerhalb zweier Jahre eingestellt. Diese Tatsache relativiert die Ziffer 97, deren Gewicht indes ein Vergleich mit der Periodika-Anzahl der ersten Jahrhunderthälfte verdeutlicht, aus der trotz eines „starken Anwachsen[s] der Buch- und Zeitschriftenproduktion“ (Jahn 2005, vii) lediglich zwei kurzlebige frankophone Zeitschriften bekannt sind.

Der seit dem ausgehenden siebzehnten Jahrhundert das europäische Geistesleben bestimmende Einfluss Frankreichs erreicht den vormals kulturell vor allem italienisch geprägten habsburgischen Hof ,erst verhältnismäßig spät, dann aber umso intensiver“ (Wagner 1961, 507) zu Beginn der Regentschaft Maria Theresias im Jahr 1740. Nachdem sich schon in den ersten Jahrzehnten des achtzehnten Jahrhunderts französische Wissenschaftler, Künstler und Architekten um Prinz Eugen, „un des vecteurs les plus efficaces de la langue et de la culture françaises“ [einer der wirkungsvollsten Vermittler der französischen Sprache und Kultur] (Teissier 1982, 217), in Wien eingefunden haben, trifft durch die Hochzeit Maria Theresias mit Franz Stephan von Lothringen 1736 dessen aus Gelehrten und Künstlern bestehende frankophone Entourage ein; durch solche Vermittlerfiguren lässt sich die französische Kultur ,nicht nur rezipieren, sondern transferieren“ (Schmale 2002, 105). Die Erziehung der Erzherzoge Joseph und Leopold in französischer Sprache und mit aufklärerischer Lektüre hat entscheidenden Einfluss auf deren politische Perspektive, dennoch folgt laut Vilain „a noticeable decline in the pre-eminence of French culture“ (Vilain 2005, 5) unter Joseph II., der anstelle der Imitation Frankreichs die Bereicherung der österreichischen Kultur durch die französische propagiert hat. Sinkt seit seinem Regierungsantritt „die Zahl der in Wien gedruckten französischen Bücher merklich“(Wagner 1961, 511), nehmen die Erstveröffentlichungen französischsprachiger Zeitschriften infolge der literaturpolitischen Liberalisierung stark zu. ${ }^{3}$

Zwischen 1750 und 1780 beläuft sich die Anzahl neuer französischsprachiger Periodika noch auf jeweils fünf bis sechs pro Jahrzehnt, darunter auch die erste Literaturzeitschrift, die Gazette françoise littéraire de Vienne (1769), die jedoch im Jahr ihres Entstehens eingestellt wird. Zwischen 1780 und 1790, in der josephinischen Ära, steigt die Anzahl drastisch auf 22 an. Die Gebrüder Gay, aus Straßburg stammende Drucker, Buchhändler und Herausgeber des von 1784 bis 1785 wöchentlich erscheinenden Journal de Vienne, dédié aux amateurs de la littérature, verleihen im ersten Heft ihrer Freude über den toleranten, fortschrittlichen und geistreichen „grand Joseph“ (Journal 1784 [Bd. 1], 29) Ausdruck, der Wis-

3 Übersicht der Anzahl an Neuerscheinungen französischsprachiger Periodika in Österreich: 1750-60: 5, 1760-70: 6, 1770-80: 5, 1780-90: 22, 1790-1800: 0, 1800-10: 21, 1810-20: 19, 182030: 6, 1830-40: 5, 1840-50: 8. 
senschaft und Literatur schütze und unterstütze. Diese aus Literaturauszügen und -besprechungen sowie Briefen, Abhandlungen und Anekdoten bestehende Zeitschrift beginnt mit einem Überblick über die Literaturgeschichte seit der Antike und widmet sich daraufhin ausführlich dem vergangenen und gegenwärtigen état des lettres in Frankreich, mit klarer Präferenz für ersteren. Als Glanzzeit der Künste wird die Epoche Ludwigs XIV. gepriesen, mit Lobreden auf Corneille, der Helden zeichne, wie sie sein sollten, auf Racines stets natürlichen und eleganten Stil, auf Molière und La Fontaine, die ohnegleichen blieben (vgl. Journal 1784 [Bd. 1], 16-17). Ganz anders die Gegenwart: In ihr seien Künste und Wissenschaften durchdrungen von der modernen Philosophie, einer fanatischen Sekte kalter und steriler Seelen (vgl. Journal 1784 [Bd. 1], 20f.). Nur der sechs Jahre vor Erscheinen der Zeitschrift verstorbene Voltaire bildet eine Ausnahme, er wird als Lyriker, Dramatiker und Epiker aufgerufen, der erheblichen Einfluss auf Geist und Sitte seines Jahrhunderts hatte, auf die Literatur allerdings einen nicht nur günstigen: Durch das Los, fast alle Literaturgattungen fabelhaft zu beherrschen, habe er diese vermengt, so dass sich in seinen Stücken pompöse Maximen (vgl. Journal 1784 [Bd. 1], 23) wiederfänden, die das nach Neuigkeiten gierende Publikum in Massen anzögen. Schlimme Folgen zeitige dabei weniger ein hervorragender Mann wie Voltaire als die mit weniger Talent ausgestatteten Epigonen, die zur Korruption des guten Geschmacks beitrügen. Mit Nachdruck kritisiert man die Ungehörigkeit und Trivialität der Boulevard-Spektakel im Stil von Beaumarchais' Stück La folle journée ou le Mariage de Figaro (1778), die geschrieben würden „pour satisfaire l'avidité stupide de tout un peuple“ [um die stumpfsinnige Gier eines ganzen Volkes zu befriedigen] (Journal 1784 [Bd. 1], 24). Der am 27. April 1784 im Pariser Théâtre Français uraufgeführte Figaro - „une monstruosité d'intrigues, d'événemens, de caractères“ [eine Monstrosität an Handlungen, Geschehnissen, Charakteren] (Journal 1784 [Bd. 1], 24) - beschäftigt das Journal de Vienne nachhaltig; nur ein Berichterstatter würdigt dabei die Neuartigkeit des Stückes: „Il semble que les auteurs comiques qui l'ont précédé, ont toujours eu l'intention de faire rire les grands aux dépens des petits; ici au contraire ce sont les petits qui rient aux dépens des grands [...]. On diroit qu'ils viennent se consoler de leur misère, en s'amusant des ridicules de ceux qui en sont les instrumens.“ [Es scheint, als ob die Komödien-Dichter, die ihm vorangegangen sind, immer die Absicht hatten, die Großen auf Kosten der Kleinen zum Lachen zu bringen. Hier dagegen sind es die Kleinen, die auf Kosten der Großen lachen [...]. Man möchte meinen, dass sie sich über ihr Elend trösten, indem sie sich über die Lächerlichkeiten derer amüsieren, die es bedingen.] (Journal, 1785 [Bd. 4], 44). Während Beaumarchais’ umfangreiche Präsenz der Aktualität geschuldet ist, findet Voltaire, bei dem es sich um den am häufigsten ins Deutsche übersetzten (vgl. Lüsebrink et al. 1997, 69-72), zugleich aber meistverbotenen Autor dieser Zeit handelt (vgl. Bachleitner 2017, 
80), in allen hier vorgestellten Zeitschriften Erwähnung. Sein Renommee gründet nicht auf seinen aufklärerischen Schriften, vielmehr wird er zum „Nestor et même le dictateur de la littérature françoise“ [Nestor und sogar Alleinherrscher über die französische Literatur] gekürt, vor allem als „l'immortel auteur de la Henriade“ [unvergänglicher Autor der Henriade] (Spectateur 1819 [Bd. 1, Nr. 6], 41).

Während das Zeitschriftenpublikum von entsprechendem Gedankengut ferngehalten wird, liest man die Schriften der Aufklärer vor allem im Adelsstand (vgl. Kreissler 1973, 21) und bei Hof. Die auf den Anregungen der französischen Aufklärer beruhenden wohlfahrtsstaatlichen Reformen sind, so Wagner, „weit über das hinausgegangen, was das Frankreich der Bourbonen geleistet hat“, paradoxerweise rüsten sie das Reich, „den Stürmen der großen Revolution von innen her Widerstand zu leisten“ (1961, 515). Nach dem Tod Josephs II. 1790 misslingt es seinem Bruder Leopold II., die kriegerischen Auseinandersetzungen im Gefolge der Revolution aufzuhalten: 1792, in seinem Todesjahr, erscheint die zuvor durch die Vermählung seiner Schwester Marie Antoinette mit Ludwig XVI. gefestigte Allianz zwischen Habsburgern und Bourbonen mit dem Ersten Koalitionskrieg endgültig als „ein gescheitertes Experiment“ (Hochedlinger 1992/93, 95). Nicht nur der Frieden, auch die Reformen haben ein Ende: Mit Leopolds Sohn Franz II. wandelt sich der aufgeklärte in einen reaktionären Absolutismus, die Hofburg zu ,einem Zentrum der gegenrevolutionären Propaganda“ (Fehrenbach 2008, 45), das revolutionäres Gedankengut durch Zensurverschärfungen bekämpft (vgl. Bachleitner 2017, Kap. 3) und „neue Maßnahmen zur Kontrolle und Überwachung von Ausländern, insbesondere der Franzosen“ (Reinalter 1980, 172) ${ }^{4}$, setzt. In einem solchen Kontext dienen Medien weniger der Beförderung als der Blockierung von Kulturimporten, was sich darin äußert, dass in der Dekade 1790-1800 keine einzige neue französischsprachige Zeitschrift erscheint. Kriegsbedingt entsteht „un violent courant francophobe“ [eine heftige frankophobe Strömung] (Teissier 1982, 224), Wagner sieht „die Vorliebe für die französische Kultur“ nun „von Mißtrauen, schließlich von Haß und Abneigung abgelöst“ (1961, 511).

Dass kultureller Transfer ,in engem Konnex mit gesellschaftlichen und politischen Macht- und Hegemonialverhältnissen“ (Suppanz 2003, 22) steht, zeigt sich neuerlich zwischen 1800 und 1810, dem Jahrzehnt der napoleonischen Belagerungen, in dem 21 neue frankophone Zeitschriften erscheinen. Die Anthologie littéraire et universelle, von 1805 bis 1806 vierzehntägig herausgegeben von dem aus Lothringen stammenden Verleger Philipp Joseph Schalbacher, setzt bewusst

$4 \mathrm{Zu}$ ihnen zählen auch die unter den Herausgebern französischsprachiger Periodika in Österreich überproportional vertretenen Verleger aus dem frankophonen Ausland (Elsass, Lothringen, Belgien), wie etwa die genannten Gay und Schalbacher (vgl. Frank und Frimmel 2008, 49-50). 
auf ein Frankreichbild aus besseren Zeiten. So wird im Porträt „Sur ce qu'on entendoit par homme aimable en France, il y a 50 ans“ [Über das, was man vor fünfzig Jahren in Frankreich unter einem liebenswürdigen Menschen verstand] der Franzose mithilfe althergebrachter positiver Stereotype in Erinnerung gerufen als „[a]mant romanesque, ami sûr et fidèle“ [romantischer Liebhaber, zuverlässiger und treuer Freund], „guerrier par honneur et par goût, philosophe sans le savoir, plein de franchise et de générosité, avec une raison supérieure“ [Krieger aus Ehre und Vorliebe, Philosoph, ohne es zu wissen, voll Offenherzigkeit und Großzügigkeit, mit überlegenem Verstand] (Anthologie 1805 [Bd. 2, Nr. 2], 128) und glühender Bewunderer der Künste. Mit der Perpetuierung eines vorrevolutionären Frankreichbildes, das trotz des veränderten politischen Klimas die Affinität zur französischen Kultur in Österreich anfachen soll, korrespondiert das geringe Verständnis für die oberflächlichen revolutionären Geister (vgl. Anthologie 1805 [Bd. 2, Nr. 3], 178), deren finsterer Atheismus deprimiere und den Menschen falsche Freiheit vorgaukle (vgl. Anthologie 1805 [Bd. 2, Nr. 3], 229), und die die Sitten, die Literatur, die Kunst, selbst die sicher geglaubte Wissenschaft verdorben hätten: So gebrauche etwa Diderot seine Geisteskräfte dazu, das Fundament aller Religionen zu unterhöhlen; da durch die schlecht ausgewählten Mitarbeiter auch die Encyclopédie trotz einiger gelungener Beiträge „une infinité de choses mal vues“ [eine Unmenge falsch verstandener Dinge] (Anthologie 1805 [Bd. 2, Nr. 2] 135) aufweise, von der Obszönität der Bijoux indiscrets nicht zu reden, werde der Nachwelt von Diderot wohl nichts erhalten bleiben. Auch von Rousseau wird in der Anthologie, die reich an Artikeln zur Erziehung und zur Moral ist, abgeraten, denn sein Émile verwirre die Phantasie und das Urteilsvermögen, huldige den Leidenschaften und verkehre die soziale Ordnung (vgl. Anthologie 1805 [Bd. 2, Nr. 2] 104).

Die 21 Publikationen zwischen 1800 und 1810 sprechen für ein Abklingen der antifranzösischen Haltung, das auch die Auseinandersetzungen mit Napoleon nicht aufhält, vielmehr beschleunigt die militärische Expansion „die grenzüberschreitende Ausbreitung von Ideen, aber auch von Handlungsmodellen (etwa im Verwaltungsbereich) und Rechtsnormen“ (Lüsebrink und Reichardt 1997, 9f.). Zur Zeit der Belagerung Wiens 1805 lässt sich laut Kreissler eine „renaissance française“ $(1973,63)$ beobachten, die er auf einen gewissen Opportunismus der Wiener zurückführt, die „toujours enclins à parler la langue du vainqueur“ [immer geneigt, die Sprache des Siegers zu sprechen] $(1973,17 \mathrm{f}$.$) seien.$ Das Französische, seit theresianischen Zeiten Diplomaten-, Hof- und Adelssprache mit Ausbreitungstendenz auf die oberen Schichten des langsam wachsenden Bürgertums, findet durch die Präsenz der französischen Truppen Eingang in den Wortschatz des Volkes, wenn auch nur durch die Verwendung einzelner französisierter Wörter. Als sich 1808 ein neuer Konflikt mit Frankreich ankündigt, ist die Bedeutung des Französischen in Wien laut Kreissler noch immer groß, vor 
allem wecke die Pressefreiheit unter der französischen Administration den Mut und „la sympathie des milieux libéraux“ [die Sympathie liberaler Schichten] (Kreissler 1973, 31, 32). Das Jahrzehnt endet, nach der zweiten Belagerung 1809, mit der im Frühjahr 1810 von Außenminister Metternich in die Wege geleiteten Verlobung der Erzherzogin Marie-Luise mit Napoleon.

Die Veröffentlichungswelle französischer Literaturzeitschriften hält mit einer Anzahl von 19 noch zwischen 1810 und 1820 an, trotz des sich verschärfenden Klimas von Verfolgung und Verboten nach Napoleons Niederlage und der Neuordnung Europas beim Wiener Kongress 1814/15. In die Restaurationszeit fällt das Erscheinen von Le Spectateur. Journal historique, littéraire, moral, politique et dramatique, zwei Mal wöchentlich von Jänner bis Juni 1819 samt unregelmäßig beigefügtem Feuilleton du Spectateur, herausgegeben von dem Komponisten und Kulturschaffenden Eduard von Lannoy und dem aus Belgien stammenden Erzieher und Übersetzer Abbé Libert. Ihnen zufolge hebt sich das unter dem Leitspruch „Vérité, impartialité“ laufende Journal von anderen dadurch ab, dass es die sonst fast immer getrennten Bereiche Geschichte, Literatur und Moral vereint und dass die vertretenen „,cosmopolites en littérature“ [Kosmopoliten in der Literatur] (Feuilleton du Spectateur 1819 [Nr. 10], 199, 200) nationale Ketten sprengen würden. Überdies kündigt der Spectateur interessante Neuerscheinungen an, doch dominieren auch hier Werke von bereits verstorbenen Schriftstellern, wie die Euvres de St. Lambert oder die Mémoires et Correspondances de Madame d'Épinay, die Details über Épinays Verbindungen mit Duclos, Rousseau, Grimm, Diderot und sonstiger Literaturprominenz des achtzehnten Jahrhunderts beinhalten. Was den Spectateur tatsächlich von den bisher angeführten Zeitschriften unterscheidet, ist die Stimme der Verfasser, vernehmbar in Äußerungen zum Zeitgeschehen und in Angriffen auf konkurrierende Blätter und Kritiker; so wird unter anderem die Wahl des Französischen verteidigt mit der Vorrangstellung der Sprache in Europa:

L'acceuil favorable que le public a fait à notre Journal a déplu à quelques envieux qui s'étonnent qu'un Journal écrit dans une langue étrangère plait à Vienne. Nous répondons à ces Messieurs que la langue française ne peut pas être nommée étrangère dans aucun Pays de l'Europe, car elle est la langue diplomatique et par cette raison la langue universelle qui doit jouir de tous les avantages dont le latin jouissait autrefois. [Die günstige Aufnahme unseres Journals durch das Publikum hat einigen Neidern missfallen, die sich darüber wundern, dass ein in einer Fremdsprache geschriebenes Journal in Wien Anklang findet. Wir antworten diesen Herren, dass die französische Sprache in keinem Land Europas als fremd bezeichnet werden kann, da sie die Sprache der Diplomatie und daher Universalsprache ist, die sich aller Vorteile erfreuen darf, derer sich das Lateinische einst erfreute.] (Feuilleton du Spectateur 1819 [Bd. 1, Nr. 12], 232)

Diese Aussage entwirft gewissermaßen ein Bild des Zielpublikums, wie ähnlich schon in den 1780er Jahren das Journal de Vienne, in dem es heißt, das Blatt sei 
„en langue étrangère, mais presqu'universellement connue“ [in einer fremden, aber fast allbekannten Sprache] (Journal 1784 [Bd. 1] 29) geschrieben. Zwar erreicht die französische Sprache bald nach dem „Höhepunkt des französischen Einflusses“ (Wagner 1961, 509) von 1750 bis 1770 ihre größte Ausbreitung (vgl. Teissier 1982, 222), allerdings nur in ,besseren' Kreisen. Die Angesprochenen bilden einen Bruchteil der Bevölkerung, von der man in ihrer Masse behaupten kann, ihr sei jeder Zugang zur Literatur und selbst zur Zeitschriften-Lektüre verwehrt (vgl. Kreissler 1973, 21).

In weiterer Folge verliert die französische Sprache durch die wachsende Bedeutung des Deutschen (vgl. Oravetz 1930, 20), die einen Aufschwung des Übersetzungswesens bewirkt, zunehmend an Wichtigkeit. Das Schwinden der frankophonen Literaturimporte ist Folge der in Auflösung begriffenen kulturellen Verflechtungen durch nationalstaatliche Bestrebungen wie auch der Ausbreitung des Lesens auf weniger gebildete Schichten. Sind es zwischen 1810 und 1820 noch 19, sinkt die Zahl der französischsprachigen Literaturzeitschriften im Verlauf des Vormärz auf nur mehr sechs zwischen 1820 und 1830, und fünf zwischen 1830 und $1840 .{ }^{5}$ Unter den acht Zeitschriften zwischen 1840 und 1850 findet sich das von Jänner bis Dezember 1841 drei Mal wöchentlich erscheinende Journal de la littérature étrangère des Herausgebers und Redakteurs Anto[i]n[e] Langerhanns. Die darin vorgestellten und erstmals überwiegend mit Verfasserangaben versehenen Werke sind recht aktuell, so z. B. Eugène Sues historischer Roman Le Colonel de Surville (1840). Die Zeitschrift befasst sich ihrem Titel gemäß mit ausländischer Literatur, d.h. neben der französischen etwa auch mit der spanischen, niederländischen und vor allem englischen, vertreten unter anderem durch Passagen aus Charles Dickens' Master Humphrey's Clock (1840). Weiters erhält die Leserschaft Einblicke in die Lebensgewohnheiten anderer Länder durch Porträts wie „Esquisse de la vie privée de Londres“, „Un dîner anglais“ oder „Antiquités skandinaves“. Neben Dumas' Reisebericht „L'été à Florence“ verdeutlichen auch Georges Sands „Le besoin de voyager“, Jules Janins „Le voyage d'un homme heureux“ und Théophile Gaultiers „Tournée en Espagne“ die Bedeutung der Reiseliteratur in einer Zeit zunehmender Mobilität. Ähnlich wie der ebenfalls kurzlebige Vorgänger dieses Organs, die Alliance littéraire (1839-1840), und der Nachfolger, die Bibliothèque instructive et récréative (1842), setzt die Zeitschrift auf längere, sich über mehrere Hefte erstreckende Literaturauszüge zuungunsten von Besprechungen und Unterhaltungsrubriken. Diese Strategie hat ebenso wenig Erfolg wie

5 Analog dazu nimmt ab ca. 1805 der Prozentsatz französischsprachiger Titel unter den in Österreich verbotenen Schriften ab (vgl. Bachleitner 2017, 165). 
der auf Sitten, Moral, Reisen und Geschichte ausgedehnte Literaturbegriff, der in den anderen hier vorgeführten Zeitschriften Anwendung fand. Letzterer führt zu einer Diversität, die in Anbetracht der großen Konkurrenz an thematisch breit aufgestellten Zeitschriften einen Nachteil dargestellt haben mag; das Hauptanliegen, die Leserschaft am aktuellen literarischen Leben Frankreichs teilhaben zu lassen, gerät so in den Hintergrund. Auch stellt der Aktualitätsanspruch aller Zeitschriften im Hinblick auf ihr häufiges Erscheinen, mit bis zu drei Heften pro Woche, eine Schwierigkeit dar, zumal fehlende Angaben zu sonstigen Mitarbeitern meist auf eine Ein-Mann-Redaktion in Person des Herausgebers schließen lassen. Da insgesamt viele Artikel französischen Periodika entnommen sind und kaum zum Lebensumfeld der österreichischen Leser in Beziehung gesetzt werden, bleibt der Mehrwert gegenüber den zahlreich aus Frankreich zu beziehenden Literaturzeitschriften fraglich. Ein Argument bietet der Spectateur, indem er sich der eigenen Unvoreingenommenheit durch den weit von Paris entfernten Standort rühmt, ,à l'abri de la cabale des auteurs, \& exempts de tout esprit de parti“ [geschützt von allen Autoren-Intrigen und frei von aller Parteinahme] (Journal de Vienne 1784 [Bd. 1], 30). Ergänzen ließe sich hier die Flucht vor der zeitweise, z. B. im vorrevolutionären Frankreich (vgl. Darnton 2014) und unter Napoleon (vgl. Horn 2013), selbst im Vergleich mit Österreich ausnehmend strengen Zensur in Frankreich.

Zwar lässt die durch die Liberalisierung des Literaturmarktes ermöglichte „floraison des gazettes littéraires“ [Aufblühen der Literaturzeitschriften] (Teissier 1982, 222) gegen Ende des achtzehnten Jahrhunderts, und vor allem die Hartnäckigkeit, mit der sich die Herausgeber der 97 frankophonen Periodika um Erfolg bemühen, auf großes Interesse an französischer Literatur schließen, doch geht dies hauptsächlich von den „oberen Gesellschaftsschichten“ (Wagner 1961, 507) aus. Es stellt sich die Frage, inwieweit innerhalb dieser des Französischen mächtigen Elite, für die auch Preisunterschiede zu in Frankreich erschienenen Literaturzeitschriften sicher nicht kaufentscheidend waren, Informationsbedarf durch in Österreich produzierte Literaturmagazine bestand, denn die rasch in Wien eintreffenden Buch-Neuerscheinungen wurden in der ,Gesellschaft' herumgereicht (vgl. Wagner 1961, 513). An sie scheinen die Inhalte der Zeitschriften indes ohnehin kaum adressiert; der implizite Leser der vorgestellten Periodika ließe sich am ehesten im mittleren bis höheren Bürgertum verorten, das zwischen 1750 und 1850 noch kein ausreichend umfangreiches Publikum dargestellt haben dürfte. Wie sich die frankophonen österreichischen Zeitschriften $\mathrm{zu}$ den in Frankreich erschienenen verhielten, von wem die Artikel in den österreichischen Periodika stammten, ob die Literaturauswahl und die Berichterstattung eine Form der Zensur darstellten oder sich eher als Flucht vor Zensur interpretieren lassen, inwieweit die häufig von den Rändern des französischsprachigen Raums kommenden Herausgeber eine für den Transfer privilegierte Position zwischen Kulturen ein- 
nahmen - diese Fragen müsste eine umfassende Erforschung des hier nur in Form von Sondierungen erschlossenen Untersuchungsfeldes beantworten.

\section{Zitierte Periodika}

Anthologie littéraire et universelle. 1805-1806.

Journal de la littérature étrangère. 1841-1842.

Journal de Vienne, dédié aux amateurs de la litterature. 1784-1785.

Le Spectateur. Journal historique, littéraire, moral, politique et dramatique. 1819.

\section{Literaturverzeichnis}

Bachleitner, Norbert. Die literarische Zensur in Österreich von 1751 bis 1848. Mit Beiträgen von Daniel Syrovy, Petr Píša und Michael Wögerbauer. Wien, Köln, Weimar: Böhlau [= Literaturgeschichte in Studien und Quellen, Bd. 28], 2017.

Darnton, Robert. Censors at Work. How States Shaped Literature. New York und London: Norton, 2014.

Fehrenbach, Elisabeth. Vom Ancien Régime zum Wiener Kongress. München: Oldenbourg [= Oldenbourg Grundriss der Geschichte; Bd. 12], ${ }^{5} 2008$.

Fichtenau, Heinrich. „Biographisches zu den Beziehungen zwischen Österreich und Frankreich im Mittelalter“. Mitteilungen des Instituts für österreichische Geschichtsforschung 70 (1962): 1-28.

Frimmel, Johannes, und Peter R. Frank. Buchwesen in Wien 1750-1850. Kommentiertes Verzeichnis der Buchdrucker, Buchhändler und Verleger. Wiesbaden: Harrassowitz, 2008 [= Buchforschung. Beiträge zum Buchwesen in Österreich; Bd. 4].

Hochedlinger, Michael. „Österreich und die Französische Revolution 1789-1792. Prolegomena zu einem Forschungsdesiderat“. Das achtzehnte Jahrhundert und Österreich. [= Jahrbuch der Österreichischen Gesellschaft zur Erforschung des achtzehnten Jahrhunderts; Band 7/8 (1992/93)]. 81-110.

Horn, Pierre. „Vom autokratischen Kaiserreich zur konstitutionellen Monarchie: Zensur und Emanzipation der französischen Presse im Vormärz (1804-1848)“. Zensur im Vormärz. Pressefreiheit und Informationskontrolle in Europa. Hg. Gabriele B. Clemens. Ostfildern: Jan Thorbecke, 2013. 23-38.

Jahn, Bruno. „Vorwort“. Die deutschsprachige Presse. Ein biographisch-bibliographisches Handbuch, Band 1, A-L. München: Saur, 2005. vii-ix.

Kreissler, Félix. Le français dans le théâtre viennois du XIXe siècle. Paris: Presses universitaires de France, 1973.

Lang, Helmut W., und Ladislaus Lang, unter Mitarbeit von Wilma Buchinger. Bibliographie der österreichischen Zeitschriften 1704-1850 [= Österreichische Zeitschriften 1704-1945;

Bd. 3]. München: Saur, 2006.

Lüsebrink, Hans-Jürgen, René Nohr, und Rolf Reichardt. „Kulturtransfer im Epochenumbruch Entwicklung und Inhalte der französisch-deutschen Übersetzungsbibliothek 1770-1815 im Überblick“. Kulturtransfer im Epochenumbruch. Frankreich-Deutschland 1770-1815. Hg. 
Hans-Jürgen Lüsebrink und Rolf Reichardt, zusammen mit Annette Keilhauer und René Nohr. Leipzig: Leipziger Universitätsverlag [= Deutsch-Französische Kulturbibliothek; Bd. 9.1], 1997. 29-86.

Lüsebrink, Hans-Jürgen, und Rolf Reichardt. „Kulturtransfer im Epochenumbruch. Fragestellungen, methodische Konzepte, Forschungsperspektiven. Einführung“. Kulturtransfer im Epochenumbruch. Frankreich-Deutschland 1770-1815. Hg. Hans-Jürgen Lüsebrink und Rolf Reichardt, zusammen mit Annette Keilhauer und René Nohr. Leipzig: Leipziger Universitätsverlag [= Deutsch-Französische Kulturbibliothek; Bd. 9.1], 1997. 9-26.

Oravetz, Vera. Les impressions françaises de Vienne (1567-1850). Paris: Presses universitaires de France, 1930.

Reinalter, Helmut. Aufgeklärter Absolutismus und Revolution. Zur Geschichte des Jakobinertums und der frühdemokratischen Bestrebungen in der Habsburgermonarchie. Wien, Köln, Graz: Böhlau [= Veröffentlichungen der Kommission für Neuere Geschichte Österreichs; Bd. 68], 1980.

Sacher-Masoch. Leopold von. Kaunitz. Kultur-historischer Roman. 3 Bde. 1: Kaunitz und Voltaire. Leipzig: Ernst Julius Günther, 1873.

Schmale, Wolfgang. „Kulturtransfer im theresianischen Zeitalter?“ Strukturwandel kultureller Praxis. Beiträge zu einer kulturwissenschaftlichen Sicht des theresianischen Zeitalters. Hg. Franz M. Eybl. [= Jahrbuch der Österreichischen Gesellschaft zur Erforschung des achtzehnten Jahrhunderts; Bd. 17 (2002)]. 95-109.

Suppanz, Werner. „Transfer, Zirkulation, Blockierung. Überlegungen zum kulturellen Transfer als Überschreiten signifikatorischer Grenzen“. Ver-rückte Kulturen. Zur Dynamik kultureller Transfers. Hg. Frederico Celestini und Helga Mitterbauer. Tübingen: Stauffenburg, 2003 [= Studien zur Inter- und Multikultur; Bd. 22]. 21-35.

Szabo, Franz A. J. Kaunitz and enlightened absolutism 1753-1780. Cambridge: Cambridge University Press, 1994.

Teissier, Philippe. „La presse de langue française éditée à Vienne au XVIIlème siècle“. Le Journalisme d'Ancien Régime. Hg. Pierre Rétat. Lyon: Presses universitaires de Lyon, 1982. 217-226.

Wagner, Hans. „Der Höhepunkt des französischen Kultureinflusses in Österreich in der zweiten Hälfte des 18. Jahrhunderts“. Österreich in Geschichte und Literatur 5 (1961): 507-517.

Wehler, Hans-Ulrich. Deutsche Gesellschaftsgeschichte; 2. Bd.: Von der Reformära bis zur industriellen und politischen „Deutschen Doppelrevolution“ 1815-1848/49. München: Beck, ${ }^{4} 2005$.

Norbert Bachleitner ist Professor für Vergleichende Literaturwissenschaft an der Universität Wien. Er hatte zahlreiche Gastprofessuren inne, unter anderem an der Sorbonne nouvelle, und ist Mitglied wissenschaftlicher Gesellschaften wie der Academia Europaea. Seine Forschungsschwerpunkte umfassen Rezeptionsforschung, die literarische Übersetzung, Buchgeschichte und Zensur sowie Intermedialität und Digitale Literatur. 
Juliane Werner ist Universitätsassistentin und Lehrbeauftragte am Institut für Europäische und Vergleichende Sprach- und Literaturwissenschaft der Universität Wien. Ihre Forschungsschwerpunkte umfassen franko-, anglo- und germanophone Kulturtransfers des 18. bis 20. Jahrhunderts, Wechselbeziehungen von Literatur und Philosophie, Word and Music Studies und Raumtheorie. 